\title{
1 The influence of saccades in shaping curved hand movement trajectory
}

2 Sumitash Jana ${ }^{1}$, Aditya Murthy ${ }^{1 *}$

$3{ }^{1}$ Centre for Neuroscience, Indian Institute of Science, Bangalore 560012, Karnataka, India

$5 \quad$ * Corresponding author:

6 Dr. Aditya Murthy

7 Centre for Neuroscience,

8 Indian Institute of Science,

9 Bangalore 560012, Karnataka,

10 India

11 Email: aditya@cns.iisc.ernet.in

12 Telephone: 080-2293-3434, Fax: 080-2293-3410

14 Running title:

15 Saccades influence curved hand trajectory

17 Keywords: sub-movements, obstacle avoidance task, eye-hand coordination, spatial coupling 18

19 Number of pages: 36

20

21 Figures: 5 
Eye and hand movements are often made in isolation but for reaching movements they are usually coupled together. While previous studies have demonstrated aspects of both kinematic and spatial coupling between eye and hand, few studies have investigated the influence of saccades on shaping a more complex curved hand movement trajectory profile. Here, using a novel obstacle avoidance task where the obstacle appeared in an infrequent number of trials, we try to establish the link between the saccade and hand trajectory. In the first part of the paper, we illustrate that the hand trajectory direction is influenced by the end location of the saccade, despite little temporal coupling between the two effectors. The $\mathrm{x}$ position of the saccade end-point was related to whether the hand trajectory followed a straight or a curved path, while the y-position of the saccade end-point was related to whether the hand took a path passing from over or below the obstacle. In the second part of the paper, we establish the link between the saccade locations and hand sub-movements and observed that the number and timing of saccades and number of hand velocity peaks were related. Taken together these results indicate that saccades can influence complex hand movement trajectories.

\section{NEW \& NOTEWORTHY}

The role of saccades on curved hand trajectory has been poorly studied. Using a novel obstacle avoidance task we were able to test this relationship during naturalistic curved hand movements. Not only were the directions of saccade end-points predictive of the direction of hand movements, there also existed a temporal relationship between saccades and hand submovements demarcated using multiple peaks in the velocity profile, demonstrating the significance of saccades in shaping complex hand trajectories. 
Our daily activities are inundated with instances of spatial coupling between eye and hand movements (Land and Hayhoe 2001; Land 2009). Despite this, it is unclear whether the eye and hand effectors share a common or separate target representation. Studies investigating eye-hand coordination have used different proxies for spatial coupling like correlations between eye and hand amplitudes, directions, end-points, and movement trajectories. In terms of amplitude correlation, some studies have suggested that a change is saccade amplitude results in a concomitant change in the hand amplitude (Bekkering et al. 1995; Kröller et al. 1999; van Donkelaar et al. 2000, 2002; de Grave et al. 2006), which has been contested by others (Pelisson et al. 1986; Binsted et al. 2001). Eye and hand end-point correlations have been found to be low by most studies irrespective of whether the targets were visible throughout the movement duration (Biguer et al. 1984; Sailer et al. 2000; Lee et al. 2014) or not (Prablanc et al. 1979), implying that eye and hand target selection are independent. Directions of eye and hand movements have also been shown to be related (Gielen et al. 1984; Soechting et al. 2001; Sailer et al. 2002; Horstmann and Hoffmann 2005; Tipper 2005), or driven by the eye system (Scherberger et al. 2003; Song and McPeek 2009; Khan et al. 2011), indicating that target selection maybe common for eye and hand. Some studies have implied that eye locations and hand trajectories are related (Neggers and Bekkering 2000, 2001; Reina and Schwartz 2003), while Stritzke and Trommershäuser (2007) has shown that eye movements are not driven by the goal of the hand movement but instead driven by low level features of the stimulus like luminance. Thus, it is still debatable whether eye and hand have common or separate target selection processes.

Furthermore, research has rarely investigated the spatial coupling between eye and hand trajectories particularly because both movements are highly stereotypical with straight movements and bell-shaped velocity profiles (Bahill et al. 1975; Morasso 1981; Flash and 
72 Hogan 1985; Collewijn et al. 1988; Harris and Wolpert 1998). However, studies have

73 demonstrated that non-normative movements like slow hand movements and curved hand

74 movements have multiple velocity peaks or sub-movements (Milner 1992; Lee et al. 1997;

75 Novak et al. 2002; Helsen et al. 2010a). Separate studies have also demonstrated that when

76 making an eye movement to a targets, subjects often make multiple saccades instead of a

77 single one (Robinson 1973; Harris 1995; Ariff et al. 2002; Berret et al. 2014). This raises the

78 interesting possibility of a spatiotemporal relationship between multiple saccades and hand

79 sub-movements which has not been investigated previously. Here, using a novel obstacle

80 avoidance task where subjects had to make curved hand movements in a fraction of trials we

81 tested the spatiotemporal coupling of eye and hand movements. Thus, we checked whether 1)

82 directions of eye and hand movements were related; 2) and whether multiple saccades and

83 hand sub-movements were related.

84 
86

\section{Subjects}

Eight subjects (all right-handed, 5 males) participated in the obstacle task. All had normal or corrected to normal visual acuity. They were aged between $24 \pm 3(\min =21$, $\max =$ 29) years. Before performing any experiment, subjects gave their written consent in accordance with the Institutional Ethics Committee of the Indian Institute of Science, Bangalore, and were paid for their participation after the conclusion of the experiment.

\section{Set-up of the experiment}

The stimuli were generated and the data was acquired at a temporal resolution of $\sim 1$ ms by TEMPO/VideoSYNC software (Reflecting Computing, St. Louis, MO, USA). A head mounted pupil tracker (ISCAN, Boston, MA, USA) was used to record eye positions at 240 Hz, while an electromagnetic tracker system (LIBERTY, Polhemus, Colchester, VT, USA) consisting of a source (central transmitter) and a tracker (small receiver) placed on the finger end point, was used to record finger positions at $240 \mathrm{~Hz}$. Both the eye and hand tracking systems interfaced with TEMPO in real time with a delay of $8 \pm 1$ ms. Fixation noise (tracker accuracy during fixation) for the eye tracker was $\sim 0.5^{\circ}$ across all the subjects (mean noise $=$ $0.51 \pm 0.11^{\circ}$, SD noise $=0.31 \pm 0.06^{\circ}$ ). Fixation noise for the hand tracker was $\sim 0.2 \mathrm{~cm}$ across all the subjects (mean noise $=0.15 \pm 0.04 \mathrm{~cm}$, SD noise $=0.12 \pm 0.03 \mathrm{~cm}$ ).

A 24” LED DELL monitor (60 Hz) was used to display the stimuli. The monitor was placed face down on a wooden frame such that the stimuli were reflected on a semitransparent mirror (25\% transmission, 75\% reflectance) placed at an angle below the monitor. Hand movements were made on an acrylic sheet placed parallel to and below the mirror. This 
set-up gave the impression that both the eye and hand movements were being made in the same plane (see Figure 1C, Gopal et al. 2015).

\section{Recording procedures}

The experiment was conducted in a dark room with subjects sitting with their chins resting at the wooden frame set-up and looking down at the mirror. The hand tracker and battery driven LED were attached on top of the pointing finger so that subjects could see their finger position in the dark. Subjects were head locked using two bars clamped to the temple to minimize head movement. A head mounted pupil tracker was placed above the left eye which captured the eye positions reflected from an infrared reflective mirror attached below the eye. Before each session began the camera was calibrated while subjects looked at targets displayed on the screen. Subjects were given $\sim 120$ practice trials before the experiment began.

\section{Obstacle task}

The task had two types of trials $-60 \%$ of the trials were no-obstacle trials and $40 \%$ of the trials were obstacle trials (see Figure 1A). Each trial began with the fixation of the eyes and finger on a central white fixation box. Following fixation duration of $500 \pm 180 \mathrm{~ms}$, a peripheral green square $\left(\sim 0.7^{\circ}\right)$ appeared at $12^{\circ}$, either to the left or right of the fixation box. In the obstacle trials, a white bar $\left(6^{\circ} \times 0.7^{\circ}\right)$ appeared between the fixation box and the target. The time between the appearance of the target and the obstacle was called the Obstacle Step Delay (OSD) and varied between 0, 83, 166, 250, and 333 ms. Additionally, the obstacle position was varied along the vertical axis (VOffset) by $\pm 0.5^{\circ}$ or $\pm 1^{\circ}$ relative to the central 
131 position. Subjects were asked to reach to the target using their right forefinger and stay there

132 till they heard a tone. Correct trials were those in which the finger reached the target within

$1331.1 \mathrm{~s}$ of the target appearance (600 ms for starting the movement and $500 \mathrm{~ms}$ to make the

134 movement), successfully circumventing the obstacle while choosing the shortest path to the

135 target. Success was indicated by auditory feedback about 200 ms after successfully reaching

136 the target. There was no explicit instruction for eye movements. Each subject performed $137 \sim 1200$ trials in two sessions with breaks being given every 50-80 trials or when he/she 138 required it.

139 Based on the type of hand movements made (refer to Figure 1A) trials were classified as:

$140 \quad$ Errors trials: If the obstacle was touched.

141 - Correct trials: If the obstacle was circumvented via the shortest path en route to the

\section{Saccade and hand movement detection}

Saccade beginning and end was marked when velocity increased above or dropped below $30 \%$, respectively. Epochs with velocity $>700 \%$ s were considered as blinks and omitted from the analysis. Saccades were further verified using the acceleration-deceleration 
154 profile, i.e. whether the peak in acceleration was followed by a peak in deceleration within 60

155 ms or less. The saccade beginning and end was adjusted using a criterion of $10 \%$ of the peak

156 velocity or $25 \%$, whichever was lesser. Finally, to be considered a valid saccade, each

157 saccade had to satisfy a duration criterion ( $>24 \mathrm{~ms} \&<140 \mathrm{~ms}$ ) and an amplitude criterion

$158\left(>2^{\circ} \&<22^{\circ}\right)$.

Hand movement beginning and end was marked when the velocity went above or

160 dropped below $10 \mathrm{~cm} / \mathrm{s}$. Movements had to satisfy an amplitude criterion $(>1 \mathrm{~cm}) \&$ a

161 duration criterion (> $40 \mathrm{~ms}$ ). Online corrected movements were detected by checking if the

162 duration was $>400 \mathrm{~ms}$ and the velocity crossed $30 \mathrm{~cm} / \mathrm{s}$ twice during the duration of the 163 movement.

\section{Cut-offs for reaction times (RT)}

RT was calculated on the basis of the detected saccades and hand movement beginnings relative to target onset, while correcting for the transmission delay of $8 \mathrm{~ms}$. For each subject, eye RT $<80 \mathrm{~ms} \&>3$ standard deviation (SD) of the Eye RT in the no-obstacle trials and Hand RT $<200$ ms \& $>3$ SD of the no-obstacle trials were removed from analysis. Less than $4 \%$ of the RT was removed using such a criterion.

\section{Angle of movements}

The saccade angle was calculated by finding the angular difference between the

174 starting point of the saccade and its end-point. The hand angle was calculated by finding the

175 angular difference between the starting point of the hand movement and the point at which

$1761 / 4$ th of the movement was completed. 


\section{Classification of online corrected trials}

180 Online corrected movements were classified into two categories: obstacle errors and

181

182

183

184

\section{Detection of minima and maxima in hand velocity profile}

To classify the eye movement angle in online corrected trials, we considered the eye angle in all the error trials in which the hand movement passed through the obstacle. If the eye angle in the online correction trial was within 2 SD of the distribution of eye angle in the error trials, the eye movement was classified as 'Eye straight'. If it the eye angle was $>2$ SD and towards the top of the obstacle it was classified as 'Eye up' and if it was $>2$ SD and towards the bottom of the obstacle then it was classified as 'Eye down'.

The minima and maxima in the hand velocity profiles were detected using the acceleration-deceleration profiles. The hand velocity profile was smoothened using a $32 \mathrm{~ms}$ sliding window. The points where the acceleration (further smoothened using a 12 ms sliding window) changed in sign was putatively marked as peaks, and the points where the deceleration changed in sign was putatively marked as dips. These dips and peaks had to be apart by a minimum of $8 \mathrm{~ms}$ and maximum of $120 \mathrm{~ms}$. Further, two peaks which were apart 
by less than $40 \mathrm{~ms}$ were also removed. This procedure resulted in a good detection of the minima and maxima in the hand velocity profiles.

\section{Target Step Reaction Time (TSRT) calculation}

As stopping is not an overt process it cannot be observed directly from the experimental results. However the race model provides a frame-work for its estimation (Logan and Cowan 1984; Camalier et al. 2007; Murthy et al. 2009; Ramakrishnan et al. 2010). This model was used to calculate the target step reaction time (TSRT) or the time taken to stop the hand movement (analogous to Stop Signal RT or SSRT, which is calculated in stop-signal paradigms). The race model considers that there exist two processes- a GO process and a STOP process which race against each other to reach a decision threshold, and the outcome of this race determines the response in an obstacle trial. If the GO process reaches the threshold first then a response to the initial target is initiated resulting in an error trial. However, if the STOP process reaches the threshold first then the response to the initial target is curbed.

The TSRT was calculated in 3 ways - mean, median, and the integration method using the logic described by Logan and Cowan (1984; see also Hanes et al., 1998; Murthy et al., 2009; Ramakrishnan et al., 2010). The first two methods assume that TSRT to be a random variable. The probability of making erroneous responses as a function of OSD (compensation function) was fitted using a cumulative weibull function and its mean (WM) was calculated. The mean TSRT was calculated as the difference between the mean no-step RT and the WM. The median TSRT was calculated using the difference between the median no-step RT and the weibull median. The integration method considers the TSRT to be a constant across all TSDs. Here, TSRT was estimated by integrating over the no-step RT until 
223 the area equalled the probability of error at that TSD, and the TSD was subtracted from this 224 value. This time was reflective of the longest GO RT which could still finish before the 225 STOP RT for that TSD and result in an error trial. The TSRT values calculated by these 3 226 methods were slightly different and hence were averaged to get a single estimate (Gopal and 227 Murthy 2016).

\section{Statistical tests}

The data was first checked for normality using Lilliefors test, and depending on its results, either a two-tailed t-test or signed rank test was used. For comparison between multiple groups, data was first checked for normality and then repeated measures ANOVA was performed. For correlations, the Pearson's correlation coefficient was used. To test if the

234 SDs of the two distributions matched, an F test was used. In the figures, to mark statistical significance the following standard will be used throughout $(p>0.05$ : NS, $p<0.05 \& p<=$ 0.01: *, $p<0.01 \& p>=0.001:{ }^{* *}, p<0.001:^{* * *}$. Cohen's $d$, which measures the effect size have also been reported. The values represented in the text represent the mean \pm SD. 
RESULTS

The eye and hand movements of 8 subjects were recorded while they performed the obstacle task where the subject had to make curved hand movements in a fraction of the trials

242 (Figure 1A), while no instruction was given for the eye movements. Subjects had to reach the peripheral target circumventing the obstacle that appeared infrequently, passing either over or below the obstacle, whichever route was shorter. The time when the obstacle appeared (Obstacle Step Delay - OSD) and the vertical position of the obstacle (VOffset) were varied. We first checked the behavioural performance of the subjects and then tested the spatial coupling between eye and hand movements.

\section{Behavioural performance}

Across the population, in the obstacle trials, correct\% was highest (46 $\pm 10 \%)$,

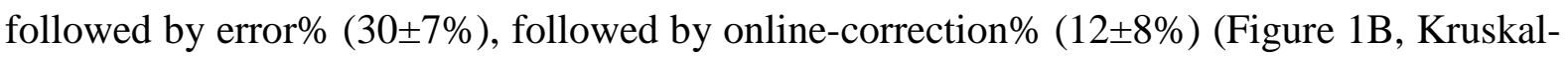
Wallis test: $\chi^{2}(2)=18.6, p<0.001$; Signed rank test: Correct $\%$ vs error $\%: W=1, p=0.016$; Error\% vs online-correction\%: $W=0, p=0.008$; Correct\% vs online-correction $\%$ : $W=0, p=$ 0.008). As expected, the error rates increased with increase in OSD (Figure 1B). Further, correct\% was positively correlated with the mean no-obstacle hand RT (Figure 1C, $\mathrm{r}=0.89$, $p=0.003$ ), indicating that when subjects took longer to initiate a hand movement they had higher chance of avoiding the obstacle if it appeared. few in number, they were related to how quickly subjects were able to stop their intended

260 movements. Using the race model framework we estimated the time taken to stop a 
movement or TSRT (Logan and Cowan 1984; Ramakrishnan et al. 2010). Thus, across subjects, online correction\% was negatively correlated with TSRT $(r=0.87, p=0.005)$.

\section{Effect of $x$-position of eye end-point on hand trajectory}

The main objective in the task was to elucidate the influence of eye position on the

266

267

268

269

270

271

272

273

274

275

276

277

278

279

280

281

282

283

284

trajectory of hand movements. We hypothesised that if the eye and hand systems have a common or interacting spatial representation then critical aspects of the hand trajectory should be predicted by the eye movement, although no explicit instructions were given for the eyes to move. In the absence of any explicit instructions given to the eye movements, subjects often made multiple saccades before onset of the hand movement. Hence, for the following analysis, the end-point (EP) of the saccade that started before the onset of the hand movement was considered. The EP of these saccades was labelled as 'eye EP before-hand movement'. We checked the influence of x-position (EyeX EP) and y-position (EyeY EP) of the eye EP on hand trajectory separately, as we believed that the x-position and y-position of the eye EP would affect the x-positions and y-positions of the hand trajectory, respectively.

As shown in figure 2A, for a representative subject the eye EP landed close to the obstacle in the correct trials, while it landed close to the target in error trials. The mean EyeX EP was significantly different between correct and error trials $(t(399)=16.2, p<0.001, d=$ 1.8). At a population level, the mean EyeX EP was significantly greater in error trials $(9.8 \pm 0.8 \mathrm{~cm})$ compared to the correct trials $(6.9 \pm 0.5 \mathrm{~cm})($ Figure $2 \mathrm{~B} ; t(7)=15, p<0.001, d=$ 4.1). To further test the influence of EyeX EP on the outcome of the trial the distribution of EyeX EP was divided into 10 bins and for each bin the probability of an erroneous response was calculated. The probability of error response increased monotonically as the EyeX EP increased (Figure 2C, repeated measures ANOVA: $F(9,63)=37.3, p<0.001$ ). These results 
indicated that the EyeX EP influenced the outcome of the trial i.e. whether the hand passed through the obstacle or took a circular path around the obstacle.

These results were however, confounded by the influence of OSD on the probability of making an error response (Figure 1C). To negate this, the distribution of EyeX EP was checked for each OSD separately. The EyeX EP in the error trials was greater than the EyeX EP in the correct trials for most OSDs across subjects (28/40, 5 OSD X 8 subjects $=40$ conditions). At a population level (Figure 2D), the mean EyeX EP in the error trials $(7.4 \pm 1.5$ $\mathrm{cm}, 6.7 \pm 0.6 \mathrm{~cm}, 9.0 \pm 1.1 \mathrm{~cm}, 10.0 \pm 0.8 \mathrm{~cm}, 10.0 \pm 1.0 \mathrm{~cm}$ for successively increasing OSD) was significantly greater than the mean EyeX EP in the correct trials $(6.6 \pm 0.5 \mathrm{~cm}, 6.4 \pm 0.2$ $\mathrm{cm}, 7.2 \pm 0.7 \mathrm{~cm}, 8.1 \pm 1.2 \mathrm{~cm}, 8 \pm 1.2 \mathrm{~cm}$ for successively increasing OSD) in last three OSDs (OSD 166: $t(7)=5.2, p=0.001, d=1.8$; OSD 250: $t(7)=6, p<0.001, d=1.7$; OSD 333: $t(7)=5.2, p=0.001, d=1.8$, all three $p$-values is less than the Bonferroni corrected $p$-value of 0.01 ). At the lower two OSDs subjects made very few errors and this could be the reason why the difference between the mean EyeX EP in the correct and error trials did not reach significance (OSD 0: $t(5)=0.9, p=0.4, d=0.7$; OSD 83: $t(7)=1.4, p=0.21, d=0.7)$. This showed that EyeX EP influenced the hand trajectory independent of OSD. In other words, closer the eye was to the target, more probable was a straight hand movement passing through the obstacle.

However, there were other confounding factors as well. For example, if the obstacle appeared shortly after the target presentation then the subjects had more time to change their hand trajectory and re-plan a correct hand movement, circumventing the obstacle. Thus, the time between the appearance of the obstacle and the movement onset, called the reprocessing time (RPT) should influence the incidence of error hand movements. When eye and hand RPT was binned, the probability of error responses decreased monotonically with increase in RPT (repeated measures ANOVA: eye RPT: $F(9)=158.8, p<0.001$; hand RPT: 
$310 F(9)=176.1, p<0.001)$, indicating the crucial role of RPT in determining the outcome of the 311 trial. other influencing factors, a logistic regression model was constructed to check which of the

314 factors contributed to the outcome. The full model had the form:

$$
\begin{aligned}
\log \left(\frac{\pi(x)}{1-\pi(x)}\right) & \\
= & \beta_{0}+(\text { EyeX EP }) \beta_{1}+(\text { Eye RPT }) \beta_{2}+(\text { Hand RPT }) \beta_{3} \\
& + \text { their interactions }+\varepsilon
\end{aligned}
$$

where $\beta$ 's represent the coefficients, $\varepsilon$ represents the error term and $\pi(x)$ refers to the probability of error response.

From this full model the non-significant terms were omitted to generate the model with least number of factors that could explain the outcome. EyeX EP was a significant factor in all 8 subjects, indicating that eye position contributes to the outcome of the trial. In order to further validate this result a linear regression model was constructed to test the role of EyeX EP and RPT on hand trajectories. Typically in correct trials the hand had a curved trajectory and in the error trials the hand had a straight trajectory, hence the angle of the hand was used as a proxy for the outcome of the trials. The full regression model had the form:

Angle of hand $=$

$\beta_{0}+($ Eye X EP $) \beta_{1}+($ Eye RPT $) \beta_{2}+($ Hand RPT $) \beta_{3}+$ their interactions $+\varepsilon$

Although the angle of the hand was not a very good proxy for the outcome of the trial

327 (as in some error trials a curved movement is made but the curvature was inadequate to 328 circumvent the obstacle) the linear regression model showed that EyeX EP was significant 
factor influencing the angle of the hand in 7/8 subjects. Taken together these analyses suggest that EyeX EP is an important factor in deciding whether a curved or straight hand movement was made to the target.

\section{Effect of y-position of eye end-point on hand trajectory}

Next the influence of the y-component of the eye EP of saccade made before-handmovement on the hand trajectory was considered. We hypothesized that the EyeY EP may predict the decision of the hand to pass from over or below the obstacle and therefore surmised that the distribution of EyeY EP would be distinct in the two cases. We plotted the EyeY EP of all the correct trials for the two conditions: hand movement from above and below the obstacle, for each of the VOffsets separately. For a representative subject the EyeY EP landed towards the top part of the obstacle when the hand moved from above the obstacle, and the EyeY EP landed towards the bottom part of the obstacle when the hand moved from below the obstacle (Figure 3A). The mean EyeY EP was different for the two hand paths, for all VOffsets for the representative subject (t-test: $p<0.001$ for all VOffsets, all $p$-values less than the Bonferroni corrected $p$-value of 0.01). For the population level analysis, we computed the difference between the mean EyeY EP when the hand moved from above and below the obstacle for each VOffset for each subject $(2.4 \pm 1.2 \mathrm{~cm}, 2.1 \pm 1.7 \mathrm{~cm}, 1.7 \pm 1.7 \mathrm{~cm}$, $1.7 \pm 1.3 \mathrm{~cm}, 1.4 \pm 1.6 \mathrm{~cm}$ for VOffsets successively from top to bottom). This difference when pooled for each VOffset was significantly greater than 0 in $4 / 5$ VOffsets (Figure $3 B$; $t(5)=$ 4.9, $p=0.005, d=3.1 ; t(6)=3.4, p=0.015, d=1.9 ; t(7)=3.6, p=0.009, d=1.8 ; t(6)=3.7$, $p=0.008, d=1.8$ for top, top-lower, middle, and bottom-upper positions, respectively; 
352 VOffset (Bottom; $t(6)=2.4, p=0.055, d=1.3$ ), suggesting that EyeY EP was indicative of 353 the hand path.

To further validate whether the EyeY EP was an indicator of the trajectory of the hand

355

356

357

358

359

360

361

362

363

364 movement a linear regression model was constructed with the angle of the hand in the correct trials (in contrast to all the trials which was considered in a previous analysis) as the dependent variable and the VOffset, Eye RPT, Hand RPT and the EyeY EP as independent variables.

The full model had the form:

Angle of hand in correct trials

$$
\begin{aligned}
& =\beta_{0}+(\text { Voffset }) \beta_{1}+(\text { Eye RPT }) \beta_{2}+(\text { Hand RPT }) \beta_{3}+(\text { EyeY EP }) \beta_{4} \\
& +\varepsilon
\end{aligned}
$$

where $\beta$ 's refers to the coefficients and $\varepsilon$ refers to the errors.

EyeY EP was a significant factor that contributed to the angle of the hand in correct trials in 7/8 subjects. Further, the mean EyeY EP was related to the route taken by the hand and was invariant to whether the trajectory decision was correct or erroneous. The EyeY EP means in the correct and error trajectory decision trials were similar, irrespective of whether the hand moved from above the obstacle $(t(7)=0.7, p=0.515, d=0.2)$, or the hand moved from below the obstacle $(t(7)=0.9, p=0.387, d=0.2)$. These results taken together suggest that EyeY EP was able to predict which route the hand would take.

The relation between EyeY EP and Eye RPT (saccade onset with respect to the appearance of the obstacle) was also tested. In other words, we asked if the location of EyeY EP at greater eye RPT could indicate the evolution of the hand trajectory. We hypothesized that at lower RPT the EyeY EP would lie close to the centre of the obstacle because the hand 
trajectory plan at that point of time was to make a straight movement towards the target, and as the RPT increases (and the hand trajectory plan progresses) the EyeY EP would move farther from the centre and towards the edges of the obstacle. As shown in Figure 3C, as RPT increased (hotter colours) the EyeY EP tended to move towards the extremities of the obstacle as seen in a representative subject. To quantify this, the eye RPT was binned into 15 bins, and for each bin the difference between the mean EyeY EP for the hand movement from above or below the obstacle was computed (Figure 3D). A least squares regression line was fitted through these points. For all the subjects the regression line had a positive slope and was significant in 6/8 subjects (Binomial test: $p=0.035$ ), and the population slope was positive and significant as well (mean slope $=0.42 \pm 0.27$; $t$-test: $t(7)=4.3, p=0.004$ ). This suggests that as Eye RPT increases the EyeY EP tends to move towards the edges of the obstacle, indicative of increased hand movement curvature, which may be visualised as a proxy of the evolving hand trajectory plan.

\section{Relationship between eye and hand in online correction trials}

There was a small fraction of online correction trials where the hand trajectory was corrected during movement execution (Figure 1B). We tested if the pattern of eye movements could predict the trajectory of hand movements in these trials. As mentioned before, online corrected hand movements were of two types: obstacle errors and trajectory errors (Figure 1A). In obstacle errors, the hand movement usually took a straight path and then diverted towards either the top or bottom of the obstacle. In the trajectory errors, the hand initially took a path from either the top or bottom of the obstacle and then diverted towards the other path. We tested if there was a relationship between straight eye movement (Eye straight) and obstacle errors; whether eye movement in the upwards direction (Eye up) resulted in an initial 
hand movement in the same direction; and whether eye movement in the downwards direction (Eye down) resulted in an initial hand movement in the same direction. The probability of eye and hand movements being made in the same direction was calculated, with the notion that no relation between eye and hand directions would predict a probability of 0.5 .

Across subjects, the probability of observing Obstacle errors in an Eye straight trial was 0.69 , which was significantly greater than $0.5(p<0.001$, as predicted by a binomial distribution), indicating that when the eye goes straight the hand tends to follow it. Similarly, $\mathrm{P}$ (Hand up|Eye up) was 0.79 , which was significantly greater than $0.5(p<0.001)$ indicating that when eye goes in the upward direction the hand follows it. There were just 6 online correction trials with initial hand movement towards the bottom of the obstacle. The $\mathrm{P}(\mathrm{Hand}$ down|Eye down) was 0.67 but did not reach significance $(p=0.1)$. This provides additional evidence of the influence of saccades on the hand trajectory.

Taken together the previous results demonstrate the spatial coupling between eye and hand movements, where the eye EP could predict the direction in which the hand moves.

Next we analysed the influence of the eye movements made during hand movements,

414 on the hand trajectory. In the correct trials, 1 or 2 saccades (1- or 2-saccade trials) were usually initiated while the hand was moving (Figure 6A, B). The onset of the saccade was locked to the time when the hand reached maximum curvature in its path (Figure 6C). The

417 time of saccade onset in 1-saccade trials and 2-saccade trials was also positively correlated 418 with the time when hand reached the maximum curvature (Figure 6D, 1-saccade: mean $\mathrm{r}=$ 
$4190.66 \pm 0.14 ; 1^{\text {st }}$ saccade of 2-saccade: mean $r=0.7 \pm 0.15 ; 2^{\text {nd }}$ saccade of 2-saccade: mean $r=$ $4200.68 \pm 0.21)$. The time-locking of the saccade onset with the time of when the hand reached 421 maximum curvature also raised the possibility of a relationship between the time of saccade 422 onset and the time when the hand velocity peaked (Figure 6A, B). Normative hand 423 movements are usually straight and have one velocity peak, but for curved hand trajectories 424 there may be multiple velocity peaks (Abend et al. 1982; Lee et al. 1997), and these are 425 thought to comprise of multiple sub-movements, with each sub-movement signified by a velocity peak (Milner 1992; Novak et al. 2002; Fradet et al. 2009). In light of the kinematic coupling mentioned before, we hypothesised that the number of sub-movements could be related to the number saccades being generated during the hand movement. To check if there existed such a relationship, a chi-square test was performed, to test if the number of saccades (1,2, or 3 saccades) and number of velocity peaks (1,2 or 3 velocity peaks) were related to each other. In 7/8 subjects the chi-square statistic was significant (Figure 5C), implying that the number of saccades and the number of hand velocity peaks were related. This interesting relationship raised the possibility that the timing of the saccade onset and the timing of hand velocity peaks may also be related.

Hence, we checked if there existed a temporal relationship between saccades and the peaks in the hand velocity. For this analysis trials where the number of saccades and the number of hand velocity peaks were the same, i.e., trials where there were 2 saccades (1 saccade during hand movement) and 2 peaks in the hand velocity profile, and 3 saccades ( 2 saccades during hand movement) and 3 peaks in the hand velocity profile, were considered. eye-hand movements, suggesting that the study of this non-normative behaviour might 442 elucidate if any link that existed between saccades and hand sub-movements. We surmised that the end of a saccade during a hand movement might indicate the end of a hand sub- 
444 movement (or the dip in the hand velocity profile). Consistent with this idea the time of 445 saccade end (of saccades during hand movement) and the time of dip in hand velocity were 446 correlated (Figure 5D; $1^{\text {st }}$ saccade and $1^{\text {st }}$ dip in hand velocity in 2-velocity peak trials: mean $\mathrm{r}$ $447=0.56 \pm 0.15, t(7)=10.7, p<0.001 ; 1^{\text {st }}$ saccade and $1^{\text {st }}$ dip in 3-velocity peak trials: mean $\mathrm{r}=$ $4480.33 \pm 0.24, t(4)=3.1, p=0.037 ; 2^{\text {nd }}$ saccade and $2^{\text {nd }}$ dip in velocity in 3-velocity peak trials $449 \quad$ (mean $\mathrm{r}=0.64 \pm 0.18, t(4)=8.2, p=0.001)$.

$450 \quad$ These results suggest that the separate hand velocity peaks may be considered as sub451 movements of the hand. The eye movements may provide some kind of spatial and/or 452 temporal cue for demarcating each sub-movement. Such a kinematic coupling further 453 reinforces the notion of an interaction between the trajectory planning of eye and hand 454 movements. 


\section{DISCUSSION}

Previous behavioural studies that have investigated the spatial aspects of eye-hand coordination have presented mixed results, with one group of studies advocating common or shared spatial representation for eye and hand movements, and another group suggesting an independent spatial representation. Using a novel obstacle avoidance task, we demonstrate the relationship between trajectory planning of eye and hand movements. Further we illustrate the relationship between saccades and hand sub-movements, which has not been studied previously. Taken together, these results indicate a shared trajectory planning of eye and hand movements.

\section{Eye-Hand interaction at the stage of trajectory planning}

Independent trajectory planning stages do not necessarily mean non-interaction

between the eye and hand systems. In this study we demonstrate that although trajectory planning stages of eye and hand seem to be different, there was weak amplitude correlation when the eye and hand moved with different amplitudes. This effect has also been reported earlier by van Donkelaar et al. (2000; 2002). Unpublished work from the lab has also demonstrated that peak eye velocity increases when subjects are instructed to make fast/slow velocity hand movements. This is surprising because peak eye velocity is thought to be controlled by the superior colliculus and brain stem (Wurtz and Optican 1994; Sparks 2002) and not under voluntary control (Segraves and Park 1993). Other studies have also reported such changes in peak eye velocity when the eye is moved along with the hand as opposed to moving alone (Snyder et al. 2002), or when the hand is loaded with weights (van Donkelaar et al. 2004). In further validation of the interaction between the trajectory planning stages of eye and hand, the eye positions were predictive of hand paths in the obstacle. Thus, there 
seems to be a kinematic coupling between the eyes and hand in terms of the saccade onset and the hand reaching the maximum curvature in its path.

\section{Shared trajectory planning of eye and hand movements}

483

484

485

486

487

488

489

490

491

492

493

494

495

496

497

498

499

500

501

502

Shared trajectory planning of the two effectors has been demonstrated by some studies. Reina and Schwartz (2003) found that in monkeys tracing an ellipse with their hand, the eye end-points tended to be located at the extremities of the ellipse and that the time of saccade onset was related to when the hand reached the maximum curvature. While these results are similar to what was observed here there are some notable differences. In contrast to their task where the hand had a constrained path, the task used here had no explicit path instruction. Further, in their task the monkeys looked at the points of maximum curvature leading to a temporal correlation. However, in this task the eyes did not always fixate at the point where the hand reached maximum curvature (as evident from the spread in EyeY EP in figure 3A) but temporal coupling was still observed. Thus, the obstacle task provided a more naturalistic study of curved hand movements where a path is not actually specified.

Although taken together the results suggest an interaction between trajectory planning for eye and hands, an alternative possibility is that coupling is not causal but rather that the eye system has access to an efference copy of the upcoming hand movement (Ariff et al. 2002). In this context Ariff et al. (2002) instructed subjects to look where their hand were in space and found that the eye was an unbiased estimator of the hand position $150 \mathrm{~ms}$ in advance. While the notion of an efference copy cannot be ruled out it appears to be unlikely to explain the velocity (unpublished work from the lab) (Snyder et al. 2002; Lee et al. 2014) and amplitude coupling observed between eye and hand movements (van Donkelaar et al. 2000, 2002), and the relationship between the saccades and hand sub-movements. If the eye 
503

504

505

506

507

508

509

510

511

512

513

514

515

516

517

518

519

520

521

522

523

524

525

526

system possesses an efference copy of the upcoming hand movement, it should be able to predict where the hand is going to be in the future, but this efference copy should not affect the saccade metrics themselves. In addition, the incorporation of a obstacle delay was a novel feature of the experiment that allowed us to assess the relationship between eye and hand kinematics in a more casual manner that has hitherto not been studied. Thus, the kinematic coupling observed here in conjunction with results from other studies suggest that interaction between trajectory planning stages might be a more prudent explanation of the observed results.

\section{Sub-movements and its relationship with saccades}

Typical fast velocity reaching movements are smooth and produce a bell shaped velocity profile (Morasso 1981; Flash and Hogan 1985) but slow hand movements and corrective movements when accuracy is specified, show multiple velocity peaks, and asymmetric non-bell-shaped velocity profiles. A number of studies have considered such deviations from the normative trajectories as individual segments or sub-movements (Abend et al. 1982; Milner 1992; Lee et al. 1997; Novak et al. 2002). Helsen et al. (2010a, 2010b) in a high amplitude reaching task found the saccades usually overshot the target which was then amended using a corrective saccade. Interestingly, such overshoot followed by small corrective movements was also observed in the movement of the finger suggesting that the sub-movements were related. Reina and Schwartz (2003) also observed a relationship between the sub-movements and saccades. Here, we show a more systematic relationship between saccades and hand sub-movements. The timing of the saccade and the time when the hand reached its maximum curvature were related although the actual locations were not. Additionally, there was dependence between the number of saccades and the number of 
527 velocity peaks seen in the hand movement. However, we failed to observe any clear spatial 528 relationship between saccades and the hand sub-movements. Although the basis of such 529 correlations is not clear, one possibility is that sub-movements may reflect the presence of 530 virtual targets/goals of the oculomotor system that define the complex non-straight line hand 531 trajectories that are executed as smaller linear segments. Such nested sub-goals within the 532 context of a single end-point goal have been observed in the evolving activity of superior 533 colliculus neurons (Bergeron et al. 2003). Further studies of the relationship between 534 saccades and hand sub-movements are required to get a deeper understanding about the 535 control of sub-movements. 
537 The study was supported by grants from the Department of Science and Technology 538 (IRPHA), Government of India, and a DBT-IISc partnership programme grant and a DBT 539 grant to AM. SJ was supported by a fellowship from the Council of Scientific and Industrial 540 Research, India and the Indian Institute of Science.

541

\section{DISCLOSURES}

543 No conflicts of interest, financial or otherwise, are declared by the authors.

544

\section{AUTHOR CONTRIBUTIONS}

546 S.J. and A.M. designed the experiments; S.J. performed the experiments and analyzed the

547 data; S.J. and A.M. interpreted the results of the experiments; S.J. prepared the figures and

548 drafted the manuscript; S.J. and A.M. edited, revised, and approved the final version of the 549 manuscript. 
REFERENCES

551

Abend W, Bizzi E, Morasso P. Human Arm Trajectory Formation. Brain 105: 331-348,

552 1982.

553

Ariff G, Donchin O, Nanayakkara T, Shadmehr R. A real-time state predictor in motor

554

control: study of saccadic eye movements during unseen reaching movements. J Neurosci 22:

555 7721-7729, 2002.

556

Bahill AT, Clark MR, Stark L. The Main Sequence, A Tool for Studying Human Eye

557 Movements. Math Biosci 24: 191-204, 1975.

558

Bekkering H, Abrams R a., Pratt J. Transfer of saccadic adaptation to the manual motor system. Hum Mov Sci 14: 155-164, 1995.

560

Bergeron A, Matsuo S, Guitton D. Superior colliculus encodes distance to target, not saccade amplitude, in multi-step gaze shifts. Nat Neurosci 6: 404-413, 2003.

562

Berret B, Bisio A, Jacono M, Pozzo T. Reach Endpoint Formation during the Visuomotor 563 Planning of Free Arm Pointing. 7814: 1-26, 2014.

564

Biguer B, Prablanc C, Jeannerod M. The contribution of coordinated eye and head movements in hand pointing accuracy. Exp Brain Res 55: 462-469, 1984.

Binsted G, Chua R, Helsen W, Elliott D. Eye - hand coordination in goal-directed aiming.

567 Hum Mov Sci 20: 563-585, 2001.

568 Camalier CR, Gotler A, Murthy A, Thompson KG, Logan GD, Palmeri TJ, Schall JD.

569 Dynamics of saccade target selection: Race model analysis of double step and search step

570 saccade production in human and macaque. Vision Res 47: 2187-2211, 2007.

571 Collewijn H, Erkelens CJ, Steinman RM. Binocular coordination of human horizontal 
saccadic eye-movements. J Physiol 404: 157-182, 1988.

573

574

575

576

577

578

579

580

581

582

583

584

585

586

587

588

589

590

591

592

593

van Donkelaar P, Lee J-H, Drew AS. Eye-hand interactions differ in the human premotor and parietal cortices. Hum Mov Sci 21: 377-386, 2002.

van Donkelaar P, Lee JH, Drew a S. Transcranial magnetic stimulation disrupts eye-hand interactions in the posterior parietal cortex. J Neurophysiol 84: 1677-1680, 2000.

van Donkelaar P, Siu K-C, Walterschied J. Saccadic output is influenced by limb kinetics during eye-hand coordination. J Mot Behav 36: 245-252, 2004.

Flash T, Hogan N. The coordination of arm movements: an experimentally confirmed mathematical model. [Online]. J Neurosci 5: 1688-703, 1985.

http://www.ncbi.nlm.nih.gov/pubmed/4020415 [27 Jul. 2016].

Fradet L, Lee G, Dounskaia N. NIH Public Access. Acta Psychol (Amst) 129: 91-100, 2009.

Gielen CCAM, van den Heuvel PJM, van Gisbergen JAM. Coordination of fast eye and arm movements in a tracking task. Exp Brain Res 56: 154-161, 1984.

Gopal A, Murthy A. A common control signal and a ballistic stage can explain the control of coordinated eye-hand movements. J Neurophysiol 115: 2470-2484, 2016.

Gopal A, Viswanathan P, Murthy A. A common stochastic accumulator with effectordependent noise can explain eye-hand coordination. J. Neurophysiol. (2015). doi: 10.1152/jn.00802.2014.

de Grave DDJ, Franz VH, Gegenfurtner KR. The influence of the Brentano illusion on eye and hand movements. J Vis 6: 727-738, 2006.

Hanes DP, Patterson WF, Schall JD. Role of frontal eye fields in countermanding saccades: 
visual, movement, and fixation activity. J Neurophysiol 79: 817-834, 1998.

595 Harris CM. Does saccadic undershoot minimize saccadic flight-time? A Monte-Carlo study.

596 Vision Res 35: 691-701, 1995.

597 Harris CM, Wolpert DM. Signal-dependent noise determines motor planning. Nature 394:

$598 \quad 780-4,1998$.

599 Helsen WF, Elliott D, Starkes JL, Ricker KL. Temporal and Spatial Coupling of Point of

600 Gaze and Hand Movements in Aiming. http://dx.doi.org/10.1080/00222899809601340. .

601 Helsen WF, Elliott D, Starkes JL, Ricker KL. Coupling of Eye, Finger, Elbow, and

602 Shoulder Movements During Manual Aiming.

603 http://dx.doi.org/10.1080/00222890009601375. .

604 Horstmann A, Hoffmann KP. Target selection in eye-hand coordination: Do we reach to

605 where we look or do we look to where we reach? Exp Brain Res 167: 187-195, 2005.

606 Khan AZ, Song J-H, McPeek RM. The eye dominates in guiding attention during

607 simultaneous eye and hand movements. J Vis 11: 9, 2011.

608 Kröller J, De Graaf JB, Prablanc C, Pélisson D. Effects of short term adaptation of

609 saccadic gaze amplitude on hand-pointing movements. Exp Brain Res 124: 351-362, 1999.

610 Land MF. Vision, eye movements, and natural behavior. Vis Neurosci 26: 51-62, 2009.

611 Land MF, Hayhoe M. In what ways do eye movements contribute to everyday activities?

612 Vision Res 41: 3559-3565, 2001.

613 Lee D, Poizner H, Corcos DM, Henriques DY. Unconstrained reaching modulates eye-

614 hand coupling. Exp Brain Res 232: 211-223, 2014. 
615 Lee D, Port L, Performance I. Manual interception of moving targets. Exp. Brain Res. .

616 Logan GD, Cowan WB. On the ability to inhibit thought and action: A theory of an act of 617 control. Psychol Rev 91: 295-327, 1984.

618 Milner TE. A model for the generation of movements requiring endpoint precision.

619 Neuroscience 49: 487-496, 1992.

620 Morasso P. Spatial control of arm movements. Exp Brain Res 42: 223-227, 1981.

621

622

623

624

625

626

627

628

629

630

631

632

633

634

635

636

\section{Murthy A, Ray S, Shorter SM, Schall JD, Kirk G, Heitz RP, Thompson KG. Neural}

Control of Visual Search by Frontal Eye Field: Effects of Unexpected Target Displacement on Visual Selection and Saccade Preparation Neural Control of Visual Search by Frontal Eye Field : Effects of Unexpected Target Displacement on Visual Selecti. : 2485-2506, 2009.

Neggers SF, Bekkering H. Ocular gaze is anchored to the target of an ongoing pointing movement. J Neurophysiol 83: 639-651, 2000.

Neggers SF, Bekkering H. Gaze anchoring to a pointing target is present during the entire pointing movement and is driven by a non-visual signal. J Neurophysiol 86: 961-970, 2001.

Novak K, Miller L, Houk J. The use of overlapping submovements in the control of rapid hand movements. Exp Brain Res 144: 351-364, 2002.

Pelisson D, Prablanc C, Goodale A, Jeannerod M. Visual control of reaching movements without vision of the limb II. Evidence of fast unconscious processes correcting the trajectory of the hand to the final position of a double-step stimulus. Exp Brain Res 62: 303-311, 1986.

Prablanc C, Echallier JF, Komilis E, Jeannerod M. Optimal response of eye and hand motor systems in pointing at a visual target. Biol Cybern 35: 113-124, 1979.

Ramakrishnan A, Chokhandre S, Murthy A. Voluntary control of multisaccade gaze shifts 
637 during movement preparation and execution. J Neurophysiol 103: 2400-2416, 2010.

638 Reina GA, Schwartz AB. Eye-hand coupling during closed-loop drawing: Evidence of 639 shared motor planning? Hum Mov Sci 22: 137-152, 2003.

640 Robinson DA. Models of the saccadic eye movement control system. Kybernetik 14: 71-83, 6411973.

642 Sailer U, Eggert T, Ditterich J, Straube A. Global effect of a nearby distractor on targeting 643 eye and hand movements. J Exp Psychol Hum Percept Perform 28: 1432-1446, 2002.

644 Sailer U, Eggert T, Ditterich J, Straube a. Spatial and temporal aspects of eye-hand 645 coordination across different tasks. Exp Brain Res 134: 163-173, 2000.

646 Scherberger H, Goodale $\mathbf{M}$ a, Andersen $\mathbf{R}$ a. Target selection for reaching and saccades 647 share a similar behavioral reference frame in the macaque. $J$ Neurophysiol 89: 1456-1466, 6482003.

649 Segraves MA, Park K. The relationship of monkey frontal eye field activity to saccade 650 dynamics. [Online]. J Neurophysiol 69: 1880-9, 1993.

651 http://www.ncbi.nlm.nih.gov/pubmed/8350128 [27 Jul. 2016].

652 Snyder LH, Calton JL, Dickinson AR, Lawrence BM. Eye-hand coordination: saccades 653 are faster when accompanied by a coordinated arm movement. J Neurophysiol 87: 2279654 2286, 2002.

655 Soechting JF, Engel KC, Flanders M. The Duncker illusion and eye-hand coordination. $J$ 656 Neurophysiol 85: 843-854, 2001.

657 Song J-H, McPeek RM. Eye-hand coordination during target selection in a pop-out visual 658 search. J Neurophysiol 102: 2681-2692, 2009. 
659 Sparks DL. The brainstem control of saccadic eye movements. Nat Rev Neurosci 3: 952$660964,2002$.

661 Stritzke M, Trommershäuser J. Eye movements during rapid pointing under risk. Vision 662 Res 47: 2000-2009, 2007.

663 Tipper SP. Reaching affects saccade trajectories. Neurobiol. Atten. (2005). doi:

664 10.1016/B978-012375731-9/50034-3.

665 Wurtz RH, Optican LM. Superior colliculus cell types and models of saccade generation. 666 Curr Opin Neurobiol 4: 857-861, 1994.

667 


\section{LEGENDS}

669 Figure 1: Task and behaviour. A) Each trial begins with subjects fixating their eyes and

670 finger on the central fixation spot. In $60 \%$ of the trials a green square appeared to the left and 671 right of the central fixation to which subjects had to make a hand movement. These trials 672 represent the no-obstacle trials. In $40 \%$ of the trials, after the presentation of the target an 673 obstacle was presented in between the fixation spot and the target after a delay called obstacle 674 step delay (OSD) of 0, 83, 166, 250, or 333 ms. Further, the presented obstacle could be 675 displaced along the vertical axis. The correct response was when the hand circumvented the 676 obstacle and reached the target via the shortest route, while error response was when the hand 677 passes through the obstacle. There were two types of online corrections: obstacle errors 678 where the hand movement initially made a straight movement towards the obstacle and then 679 diverted towards either the top or bottom of the obstacle; trajectory errors where the hand 680 initially took a path towards either the top or bottom of the obstacle and then diverted towards 681 the other direction. B) Bar plot showing the percentage of correct (green), error (red) and 682 online correction (blue) trials. Each dot represents a subject, while the bar and cross-hair marks the mean \pm s.e.m. across the population. Correct\% was highest, followed by error\% and

684 then by online-correction\%. C) The Probability of error called the compensation function 685 increased as a function of OSD. The compensation function of each subject is represented 686 using a dotted line and the mean across the population is denoted by a thick line. D) Scatter 687 plot showing the linear relationship between the mean no-obstacle hand RT and correct\%. Each dot and cross-hair denote the mean \pm s.e.m. of no-obstacle hand RT.

Figure 2: Influence of EyeX End Point (EP) on hand trajectory. A) Eye EP for correct (green dots) and error (red dots) obstacle trials for a representative subject. The data for both the 
692

693

694

695

696

697

698

699

700

701

702

703

704

705

706

707

708

709

710

711

712

713

714

715

716

right and left targets has been pooled together. The small square at $0^{\circ}$ and the small blue square at $12^{\circ}$ represent the fixation box and the target respectively. The larger squares represent the electronic windows (a region that is specified in the behavioral monitoring system but unknown to the subject). The EyeX EP is closer to the target in the error trials. B) The EyeX EP in correct versus error trials for all subjects. Each purple circle and cross-hairs represents the mean \pm s.e.m. of a subject while the filled square and cross-hairs represent the group mean \pm s.e.m. The black dotted line represents the unity line. In correct trials the mean eyeX EP is closer to the obstacle while it lies closer to the target in error trials. C) P(Error) as a function of EyeX EP for all subjects. Each dotted line represents the Weibull fit for a subject while the solid line represents the fit across the population. D) The mean EyeX EP for correct (green dots) and error (red dots) obstacle trials for each OSD for all the subjects. The green and red squares and cross-hairs represent the group mean \pm s.e.m. of EyeX EP for the correct and error trials.

Figure 3: Influence of EyeY End Point (EP) on hand trajectory. A) The EyeY EP for correct trials for each VOffset in a representative subject. The dotted rectangles denote the location of the obstacle. The orange dots represent the EyeY EP in each trial when the hand passed from below the obstacle while the mauve dots represent the Eye Y EP when the hand passed from above the obstacle. The square and cross-hair represent the mean \pm s.e.m. of the EyeY EP. The eyeY EP largely mirrors the path that the hand took. B) Difference between mean eyeY EP for hand movement passing over the top and bottom of the obstacle for each VOffsets. Each dot represents this difference for a subject for a VOffset. The bar and crosshair represents the mean \pm s.e.m across the population. C) The EyeY EP for correct trials in the representative subject for the different VOffsets colour coded for eye RPT. The colour becomes hotter as the eye RPT increases. The circles represent the EyeY EP when the hand 
passed from over the obstacle and the squares represent the EyeY EP when the hand passed

718 from below the obstacle. D) Absolute difference between mean EyeY EP when hand passed

719 from above and below the obstacle for each RPT bin. The mauve circles and crosshairs

720 represent the difference for each RPT bin for a subject and the mauve line represents the

721 least-squares fit. The violet circles and crosshairs, and line represent the population

722 mean \pm s.e.m. of the difference and its least-squares fit respectively.

724 Figure 4: Relationship between saccade onset and maximum curvature. A) Example of a trial with a single saccade during hand movement. B) Example of a trial with two saccades during hand movement. C) Population data for the probability of saccade with respect to the time when the hand reaches the maximum curvature. Each coloured line and the shaded region represent the mean \pm s.e.m. of probability of saccades across the population; the green line represents the first saccade of 1-saccade trials, the violet line represents the first saccade of 2saccade trials and the brown line represents the second saccade of 2-saccade trials. D) Correlation between the time when hand reaches maximum curvature and the time of saccade onset in 1-saccade-during-hand trials (green), and onset of the $1^{\text {st }}$ saccade of 2-saccadeduring-hand trials (violet) and the onset of the $2^{\text {nd }}$ saccade of 2-saccade-during-hand trials (brown). The dots represent individual subjects while the bars and cross-hairs represent the mean \pm s.e.m. across the population.

Figure 5: Relationship between saccades and hand sub-movements. A) Example of a velocity profile with 2 velocity peaks (marked by pink dotted lines). The dip in the velocity is marked by dotted green line. B) Example of a velocity profile with 3 velocity peaks. The dips in the velocity are marked by dotted orange lines. C) Chi-square statistic for number of saccades 
741 and number of velocity peaks for all the subjects (dots). Filled dots denote significance. The

742 bar and cross-hair denotes the mean \pm s.e.m. of the chi-square statistic. D) Correlation between

743 the time of $1^{\text {st }}$ saccade and dip in hand velocity in 2 velocity peak trials (green), time of $1^{\text {st }}$

744 saccade and $1^{\text {st }}$ dip in the hand velocity in 3-velocity-peak trials (violet) and time of $2^{\text {nd }}$

745 saccade and $2^{\text {nd }}$ dip in hand velocity in 3-velocity-peak trials. The dots represent individual

746 subjects and the bars and cross-hairs denote the mean \pm s.e.m. across the population. 
A
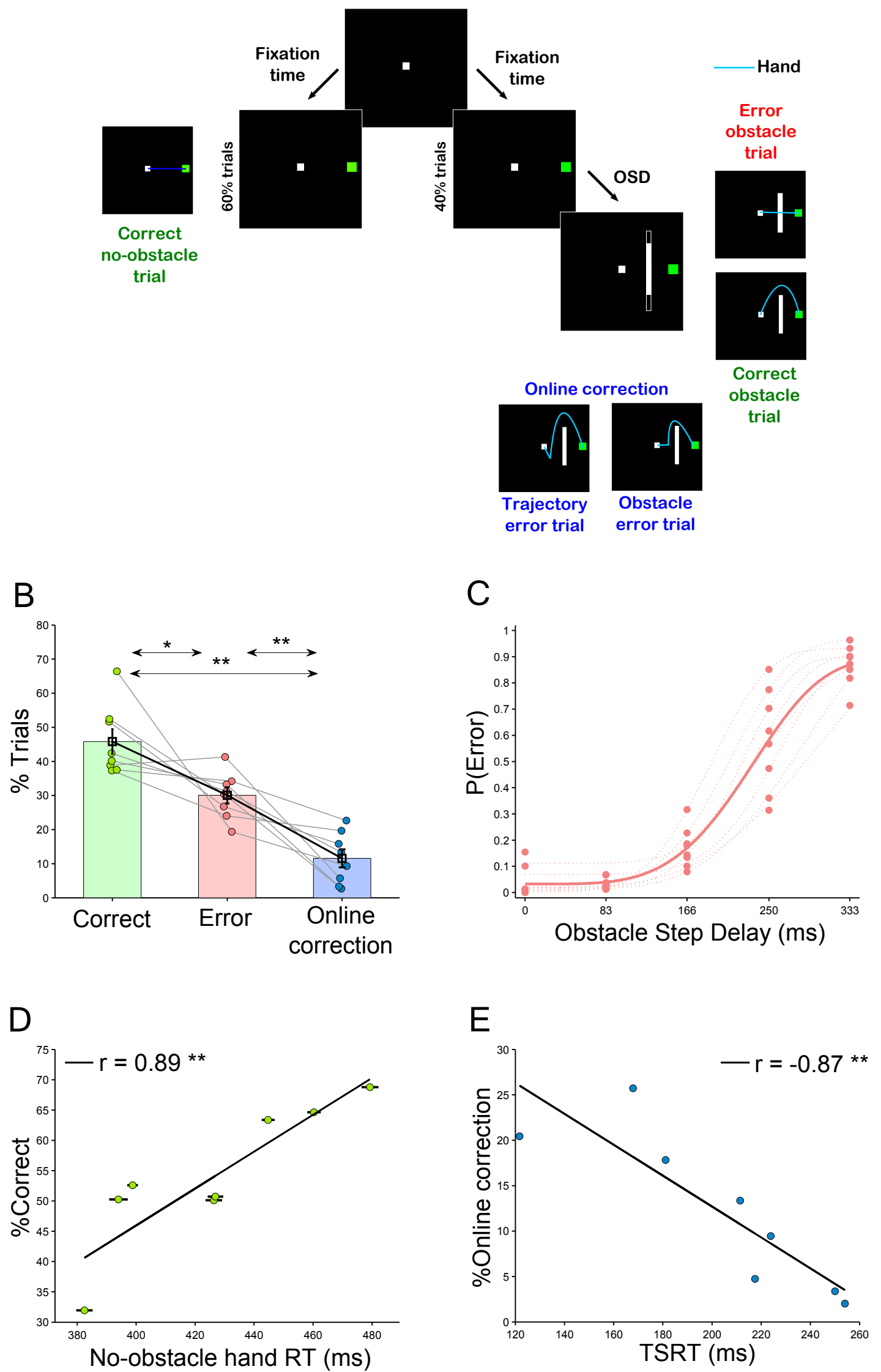

Figure 1 


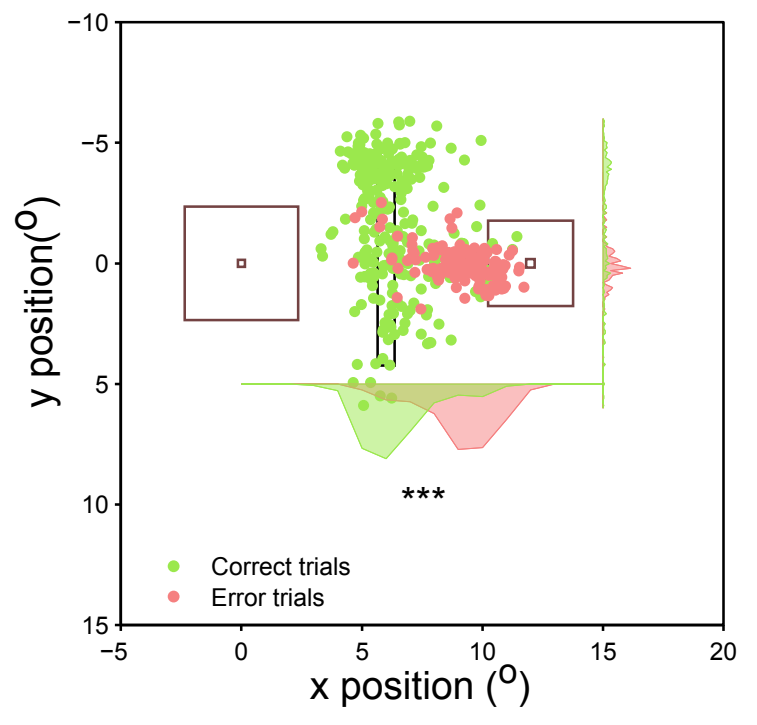

C

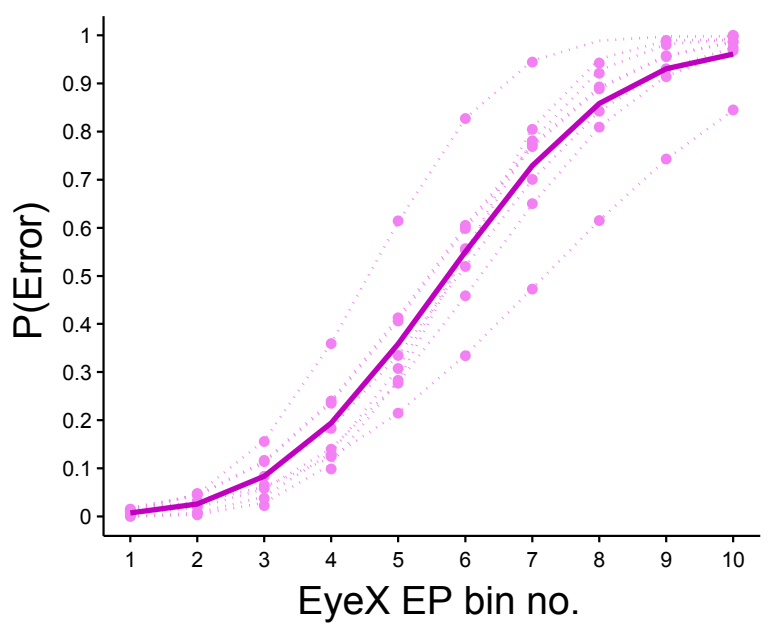

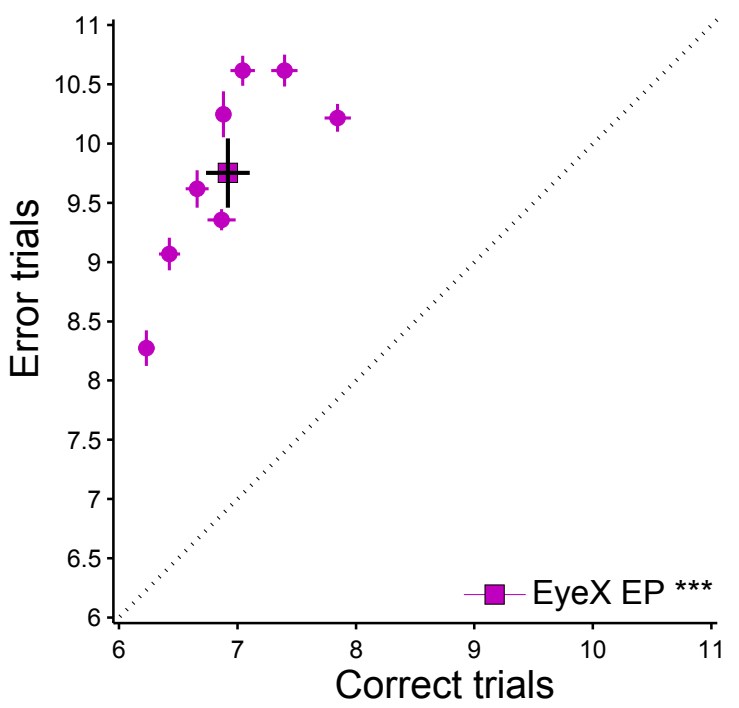

D

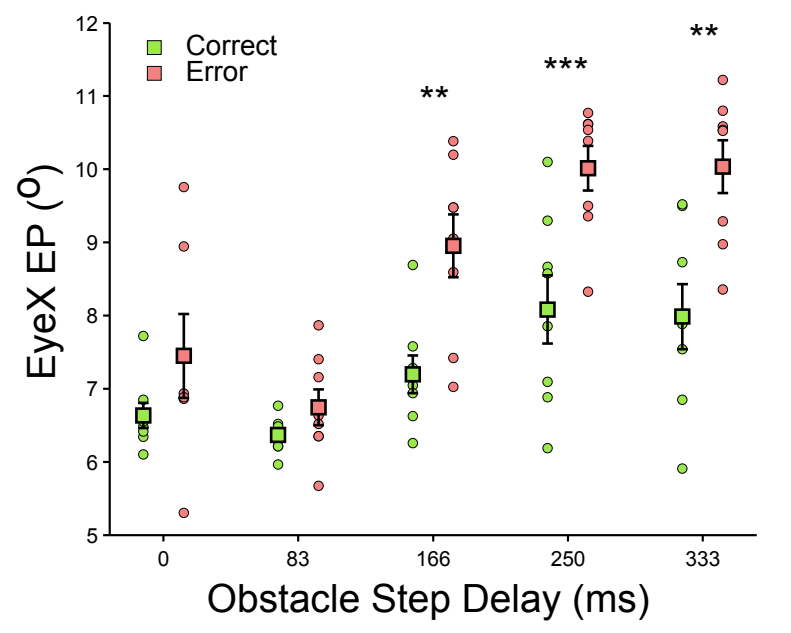

Figure 2 

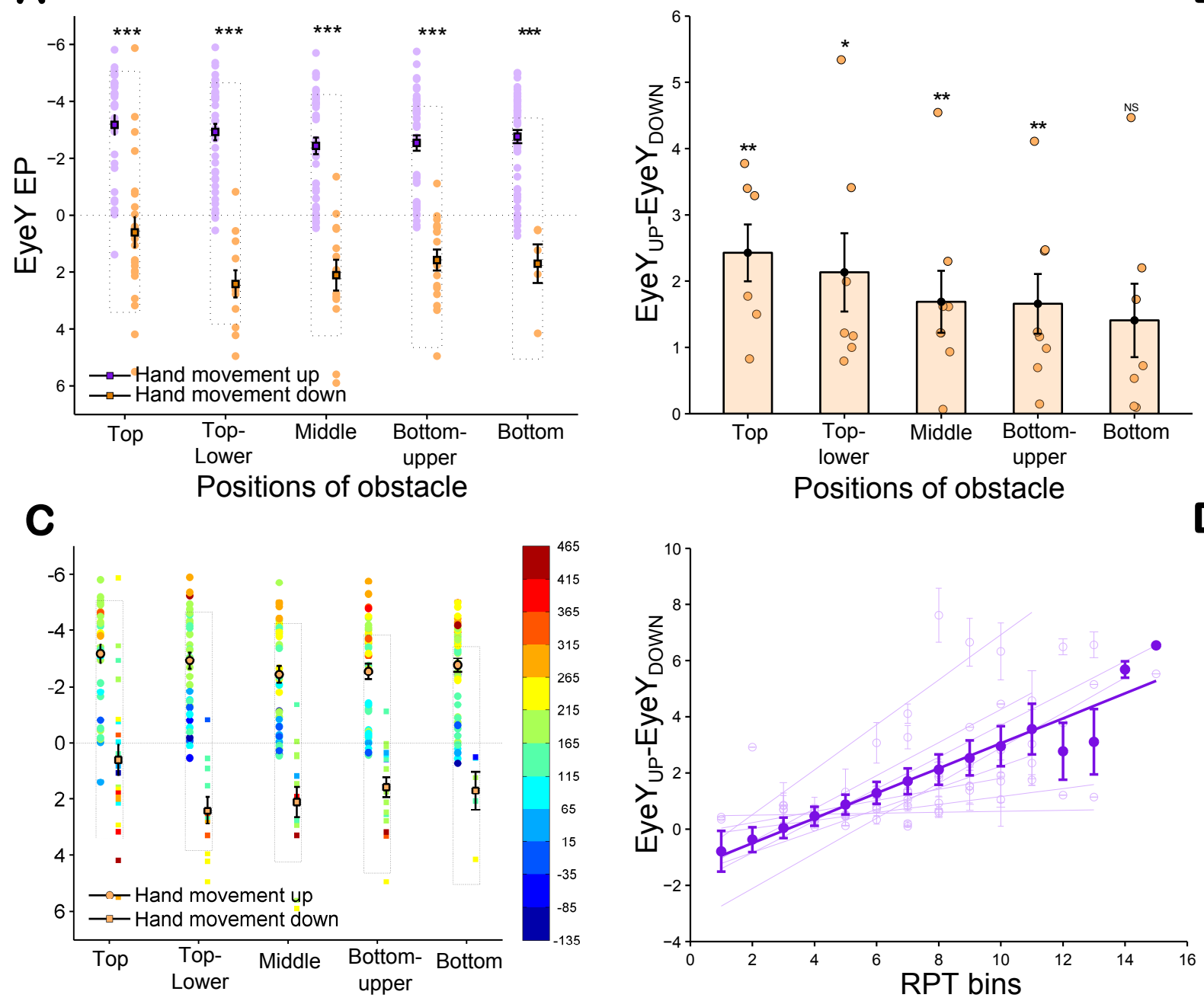

Positions of obstacle

Figure 3 

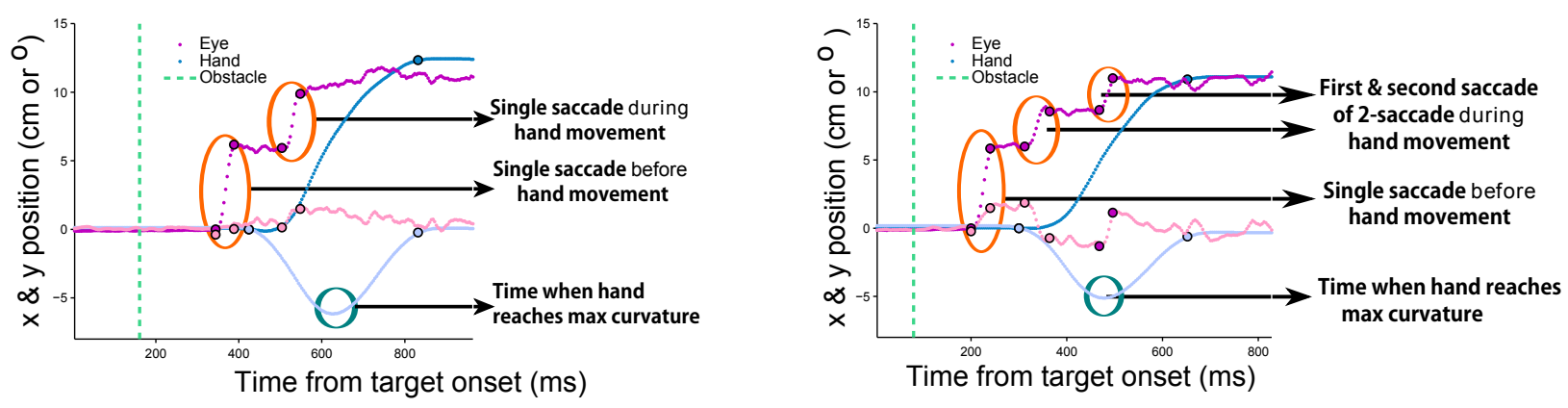

C
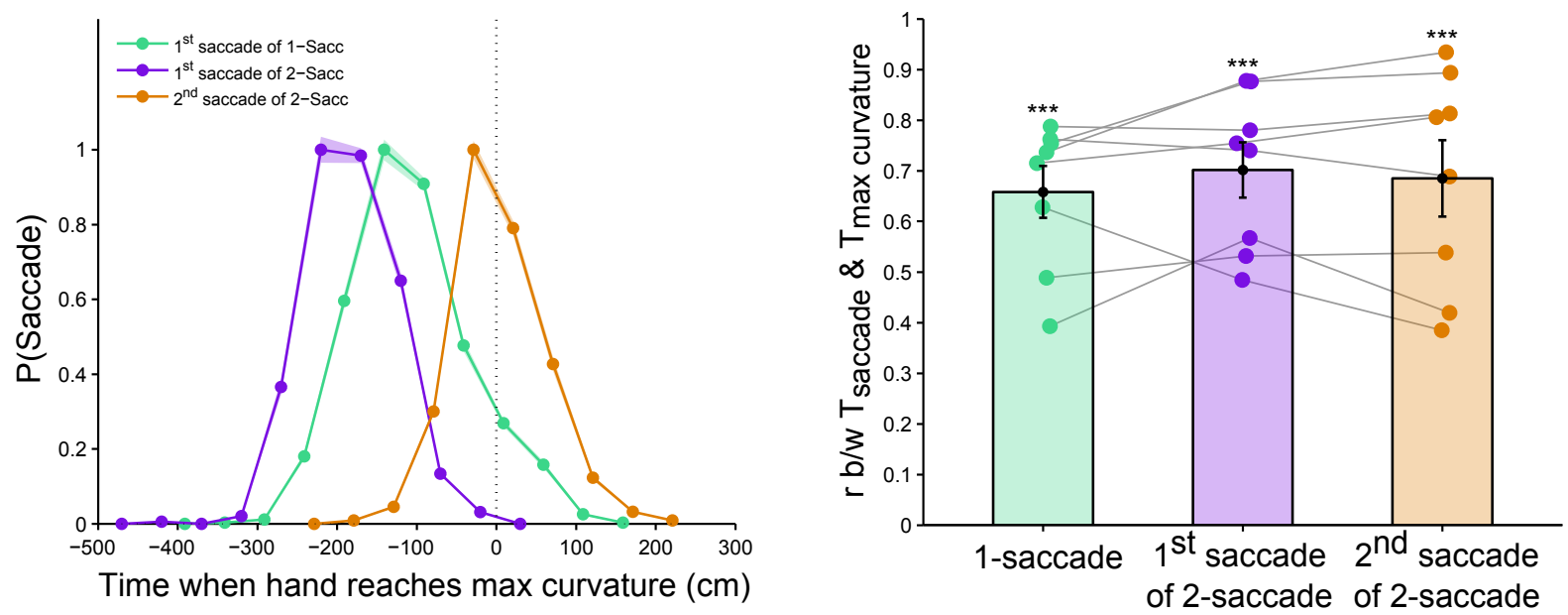

Figure 4 
A

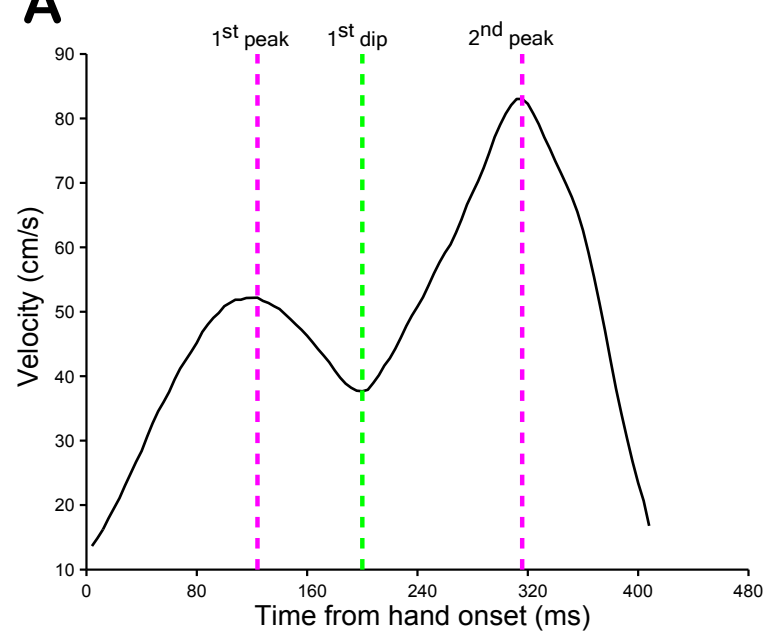

C

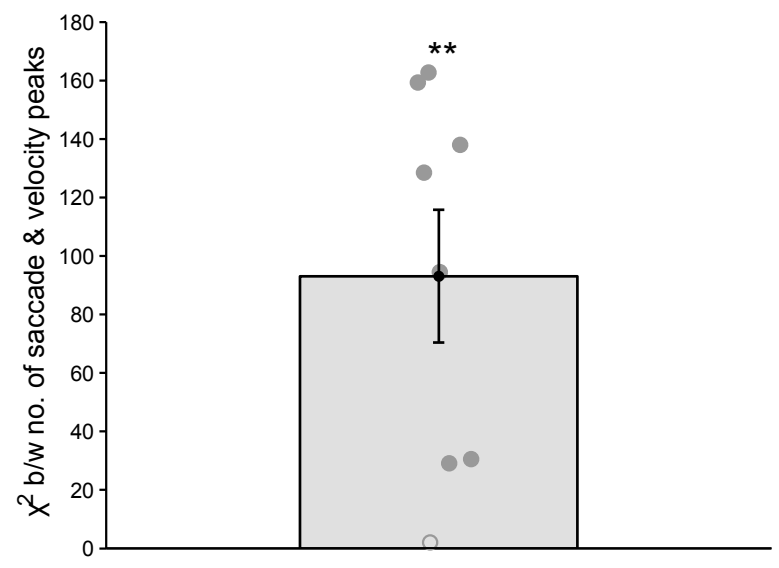

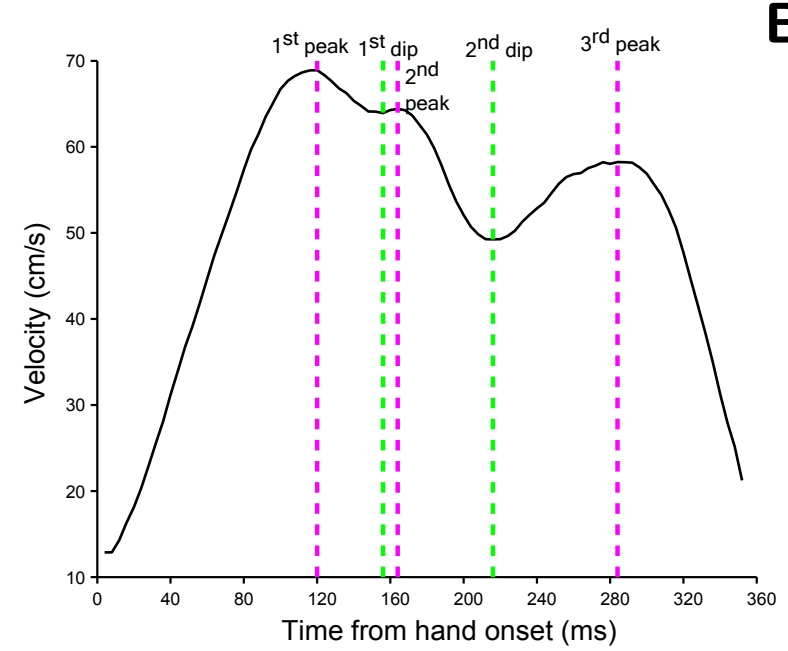

D

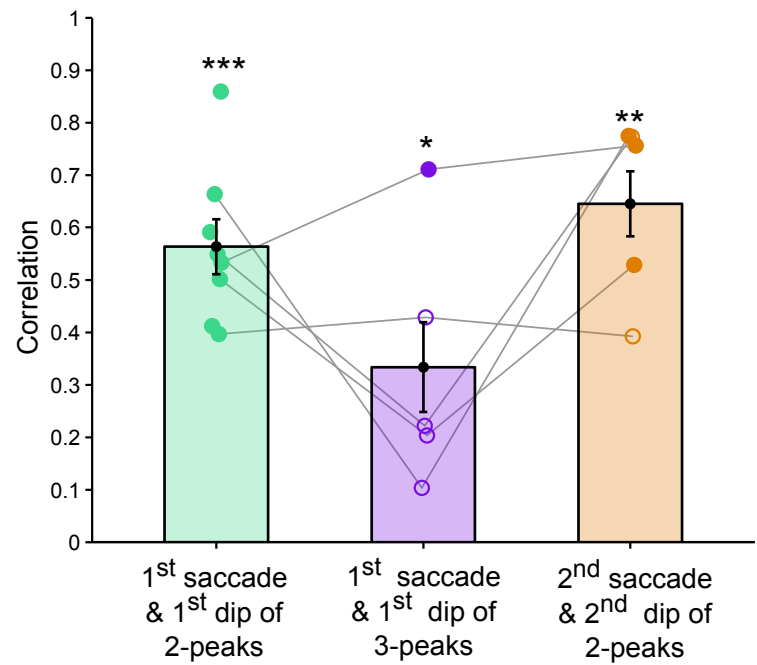

Figure 5 